\title{
Cause of recurrent laryngeal nerve paralysis following esophageal cancer surgery and preventive surgical technique along the left recurrent laryngeal nerve
}

\author{
Hiroyuki Kobayashi, Masato Kondo, Ryosuke Kita, Hiroki Hashida, Keiichi Shiokawa, Kentaro Iwaki, \\ Hiroyuki Kambe, Ryosuke Mizuno, Takuma Kawarabayashi, Tokihiko Sumi, Satoshi Kaihara, Ryo \\ Hosotani \\ Department of Surgery, Kobe City Medical Center General Hospital, Kobe City 650-0047, Japan.
}

Correspondence to: Dr. Hiroyuki Kobayashi, Department of Surgery, Kobe City Medical Center General Hospital, 2-1-1 Minatojma-minamimachi, Chuo-ku, Kobe City 650-0047, Japan. E-mail: hiro64151@kcho.jp

\begin{abstract}
How to cite this article: Kobayashi H, Kondo M, Kita R, Hashida H, Shiokawa K, Iwaki K, Kambe H, Mizuno R, Kawarabayashi T, Sumi T, Kaihara S, Hosotani R. Cause of recurrent laryngeal nerve paralysis following esophageal cancer surgery and preventive surgical technique along the left recurrent laryngeal nerve. Mini-invasive Surg 2020;4:30.
\end{abstract}

http://dx.doi.org/10.20517/2574-1225.2020.12

Received: 16 Jan 2020 First Decision: 20 Mar 2020 Revised: 2 Apr 2020 Accepted: 8 Apr 2020 Published: 16 May 2020

Science Editor: Itasu Ninomiya Copy Editor: Jing-Wen Zhang Production Editor: Tian Zhang

\begin{abstract}
Aim: Recurrent laryngeal nerve paralysis (RLNP) after esophageal cancer surgery, especially on the left, is a major clinical challenge. We believe that the use of intra-operative neural monitoring can help us to learn and identify surgical maneuvers that can cause RLNP, so as to improve the postoperative course for patients. Thus, the aim of this study was to determine the causes of RLNP and to devise a preventive surgical technique.
\end{abstract}

Methods: Radical esophageal cancer surgery was performed with intra-operative neural monitoring at our institution from July 2015 to January 2019. The cause(s) of RLNP was investigated by video analysis, which enabled a preventive technique to be developed and introduced. Short-term surgical outcomes of the modified and conventional surgical methods were compared.

Results: RLNP occurred in 10/57 (17.5\%) of cases. The causes of paralysis were traction $(n=5)$, compression $(n=3)$, thermal injury $(n=1)$, and compression in cervical procedure $(n=1)$. Subsequently, 20 surgeries were performed between February and December 2019 using the modified technique and there was only one case (5\%) of RLNP.

Conclusion: The main causes of RLNP are compression and traction. Our modified technique for esophageal cancer surgery substantially decreases the incidence of RLNP post-operatively.

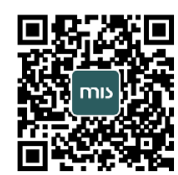


Keywords: Minimally invasive surgery, thoracoscopic esophagectomy, recurrent laryngeal nerve paralysis

\section{INTRODUCTION}

Surgery for thoracic esophageal cancer has a high rate of postoperative complications ${ }^{[1-3]}$ including recurrent laryngeal nerve paralysis (RLNP), which can lead to aspiration pneumonia and voice hoarseness, and greatly affects the postoperative course. Inflammatory complications can also affect the patient's longterm prognosis ${ }^{[4,5]}$. Of note, RLNP occurs more frequently on the left, which adds to the clinical challenge.

As such, we introduced intraoperative neural monitoring (IONM) in 2015 and have previously reported on its success in reducing the incidence of RLNP at our hospital ${ }^{[6]}$. This can be attributed to three factors: (1) mapping of the recurrent laryngeal nerve (RLN) location; (2) RLN path navigation; and (3) learning effect. Nevertheless, RLNP has not been eliminated completely, largely because the type(s) of surgical maneuver that is responsible is not known.

We hypothesized that with IONM and intra-operative video analysis, the surgical maneuver leading to postoperative RLNP can be identified. Herein, we report our findings from our experiences in applying IONM to esophagectomy. We also describe a modified procedure to prevent RLNP, especially on the left, and the short-term surgical results.

\section{METHODS}

\section{Patients}

Seventy-seven consecutive patients who underwent prone esophagectomy with radical lymph node dissection at our institution from July 2015 to December 2019 were identified. Of 57 cases treated up to January 2019, 10 (17.5\%) developed RLNP and were subjected to detailed video analysis and a preventive surgical technique for RLNP was developed. Since RLNP on the right hardly occurred, we focused on the left. Patients were divided into two groups: conventional surgery (July 2015 to January 2019, $n=57$ ) and modified surgery (February 2019 to December 2019, $n=20$ ) and short-term surgical outcomes were compared. Cancer staging was performed preoperatively according to the 8th edition of the American Joint Committee on Cancer Staging Manual by endoscopy, enhanced computed tomography, and positron emission tomography ${ }^{[7]}$. Postoperative RLNP was evaluated by laryngoscopy on postoperative day 7 . We also recorded Clavien-Dindo Grade 2 and higher complications such as aspiration, pneumonia, and anastomotic leakage. In all cases, the first author (Kobayashi $\mathrm{H}$ ), who had performed more than 100 thoracoscopic esophagectomies prior to this study, performed or supervised the surgery. This study was approved by the institutional review board.

\section{IONM and modified surgical technique}

The use of IONM has been described previously ${ }^{[6]}$. The modified surgical technique is described in the Result section. Briefly, patients were positioned prone and an electromyographic tracheal tube (Medtronic, Jacksonville, FL, USA), one-lung ventilation with blocker, no muscle relaxation, and NIM Nerve Monitoring System 3.0 (Medtronic) were used. The RLN in the thoracic cavity was localized and confirmed by IONM. At the end of the surgery, the vagal nerve was stimulated with a probe to confirm nerve functioning. Video analyses was performed by the first (Kobayashi H) and second authors (Kondo $\mathrm{M}$ ) independently. When there was concordance between assessment by the two authors, that particular maneuver would be considered the cause of RLNP.

\section{Statistical analyses}

Statistical analyses were performed using JMP version 12.0 software (SAS Institute Inc., Cary, NC, USA). Categorical variables were reported as absolute values and percentages and continuous variables are 
Table 1. Patient characteristics and surgical outcomes after technique modification

\begin{tabular}{|c|c|c|c|}
\hline & Conventional group $(n=57)$ & Modified group $(n=20)$ & $P$ value \\
\hline $\begin{array}{l}\text { Gender } \\
\text { Male:Female }\end{array}$ & $42: 15$ & $15: 5$ & 1.00 \\
\hline $\begin{array}{l}\text { Age } \\
\text { Median (IQR) }\end{array}$ & $67(17)$ & $64(16)$ & 0.71 \\
\hline $\begin{array}{l}\text { Body mass index } \\
\text { Median (IQR) }\end{array}$ & $21.3(3.5)$ & $21.0(5.6)$ & 0.53 \\
\hline Smoking yes/no & $43 / 14$ & $12 / 8$ & 0.19 \\
\hline Alcohol yes/no & $50 / 7$ & $17 / 3$ & 0.75 \\
\hline $\begin{array}{l}\text { Location of the main tumor } \\
\text { Ce:Ut:Mt:Lt:Ae }\end{array}$ & 1:8:16:31:1 & $0: 3: 5: 12: 0$ & 0.85 \\
\hline $\begin{array}{l}\text { Pathological type } \\
\text { SCC:AC:others }\end{array}$ & $50: 5: 2$ & 20:0:0 & 0.32 \\
\hline $\begin{array}{l}\text { Prior treatment } \\
\text { NAC:CRT:ESD:none }\end{array}$ & 26:1:4:26 & 10:1:1:8 & 0.22 \\
\hline $\begin{array}{l}\text { AJCC Stage } \\
\text { I:II:III:IV }\end{array}$ & 18:19:19:1 & $3: 6: 6: 2$ & 0.23 \\
\hline Thoracoscopic:Robotic:Open & $44: 13: 0$ & $0: 20: 0$ & $<0.0001$ \\
\hline $\begin{array}{l}\text { Operative time }(\mathrm{min}) \\
\text { Median (IQR) }\end{array}$ & $295(71)$ & $266(62)$ & 0.04 \\
\hline $\begin{array}{l}\text { Bleeding }(\mathrm{mL}) \\
\text { Median (IQR) }\end{array}$ & $150(218)$ & $91(255)$ & 0.08 \\
\hline $\begin{array}{l}\text { Number of dissected mediastinal LNs } \\
\text { Median (IQR) }\end{array}$ & $24(13)$ & $29(12)$ & 0.04 \\
\hline Postoperative left RLNP (laryngoscopy) & $10(17.5 \%)$ & $1(5.0 \%)$ & 0.27 \\
\hline Aspiration (CD2 and over) & $2(3.5 \%)$ & $1(5.0 \%)$ & 1.00 \\
\hline Pneumonia (CD2 and over) & $5(8.8 \%)$ & $4(20.0 \%)$ & 0.14 \\
\hline Anastomotic leakage (CD2 and over) & $9(15.8 \%)$ & $2(10.0 \%)$ & 0.32 \\
\hline $\begin{array}{l}\text { Postoperative hospital stay (days) } \\
\text { Median (IQR) }\end{array}$ & $22(17)$ & $22(7)$ & 0.82 \\
\hline
\end{tabular}

IQR: interquartile range; Ce: cervical esophagus; Ut: upper thoracic esophagus; Mt: middle thoracic esophagus; Lt: lower thoracic esophagus; Ae: abdominal esophagus; SCC: squamous cell carcinoma; AC: adenocarcinoma; NAC: neoadjuvant chemotherapy; NACRT: neoadjuvant chemoradiotherapy; ESD: endoscopic submucosal dissection; AJCC: American Joint Committee on Cancer; LNs: lymph nodes; RLNP: recurrent laryngeal nerve paralysis; CD: Clavien-Dindo grade

presented as median and interquartile range. Differences in frequency of categorical variables were assessed with Pearson's chi-squared test or Fisher's exact test, whereas continuous variables were evaluated with the Mann-Whitney two-sample statistic as appropriate. $P$-values $<0.05$ were considered statistically significant.

\section{RESULTS}

\section{Detection of RLNP}

Table 1 shows patients characteristics and short-term surgical results. There were 10 cases (17.5\%) of RLNP diagnosed by laryngoscopy on post-operative day 7. The cause of RLNP was determined by video analysis. Typical causes of RLNP identified are shown in Figure 1. Table 2 lists all causes in the 10 cases of RLNP in this study. One of the cases was excluded because the RLNP was thought to be caused by direct nerve compression during cervical procedures. In the other nine, RLNP was found to be caused by traction in the thoracic cavity $(n=5)$, compression $(n=3)$, and thermal injury $(n=1)$.

In terms of the reliability of IONM, the sensitivity (confirmed positive by IONM among verified cases of RLNP) and specificity (negative by IONM among cases of no RLNP) were 80\% (8/10) and 95.7\% (45/47), respectively [Table 3]. The positive predictive value (percentage of postoperative RLNP cases among RLNP cases estimated by IONM) and negative predictive value (percentage of no postoperative RLNP among patients with no RLNP estimated by IONM) were 80\% (8/10) and 95.7\% (45/47), respectively.

\section{Surgical technique}

Based on the above results, we modified our surgical procedure to try to avoid RLNP. To counter thermal injury, it is necessary to identify the location of the RLN in advance by using IONM as before. We 

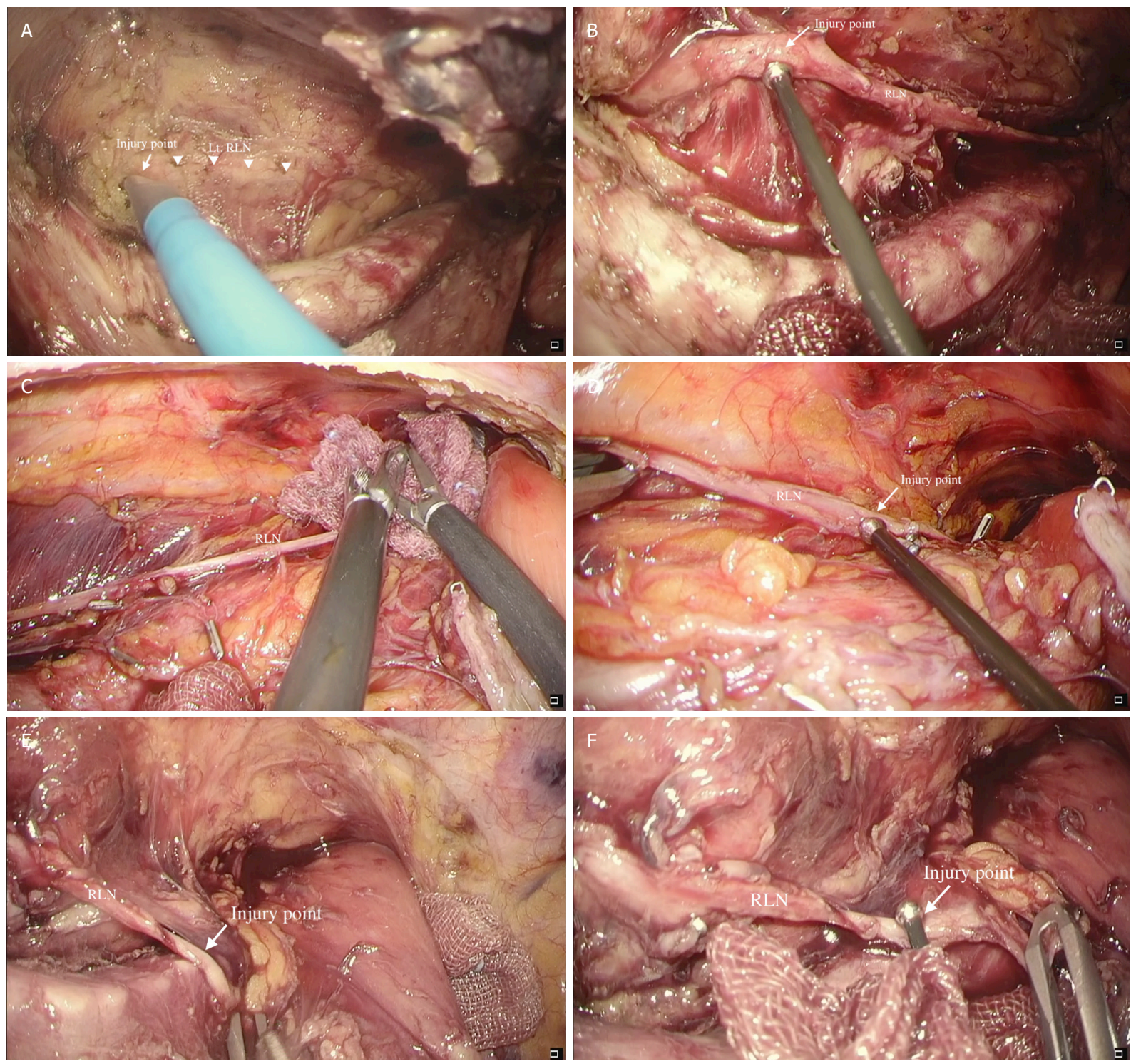

Figure 1. Learning from IONM. The causes of RLNP were revealed by intra-operative video analysis. $A$, $B$ : in the first case, the electric scalpel touched the RLN before it was recognized (A), resulting in a loss of signal at this point (B). Thermal damage was deemed to be the cause of paralysis in this case. C, D: in the second case, the surgeon compressed the area around the RLN to achieve hemostasis (C). Signal loss occurred at this point (C) and compression of the nerve between the forceps and trachea was determined as the cause of paralysis. E, F: in the third case, excessive traction damaged the RLN at the edge of trachea (E), leading to signal loss. IONM: intraoperative neural monitoring; RLN: recurrent laryngeal nerve; RLNP: recurrent laryngeal nerve paralysis

preferred to use a bipolar device that did not heat up excessively and thus, reduce the spread of heat to the surroundings compared to other energy devices such as laparoscopic coagulating shears. We also avoided traction and compression during the surgery by moving the RLN as little as possible [Figure 2]. The procdure was modified for the left side only; for the right side, we made no changes to the previously reported method $^{[6]}$. A supplemental intraoperative video is available.

The esophagus was first severed at the level of the aortic arch, peeled towards the cranial side, and the stump was sewn to the chest wall to expand the operative field [Figure $2 \mathrm{~A}$ ]. We then compressed the trachea to further expand the field of view, and started dissecting lymph nodes around the left RLN [Figure $2 \mathrm{~B}$ ]. Using the glossy membrane as a landmark of the ventral limit of lymph node dissection, we peeled away the adipose tissue, which included the lymph nodes to be dissected [Figure $2 \mathrm{C}$ and D]. Next, we removed the 
Table 2. Causes of postoperative recurrent laryngeal nerve paralysis

\begin{tabular}{ll}
\hline Stretch/Traction & 5 \\
Compression & 3 \\
Thermal/Heat injury & 1 \\
Ligature & 0 \\
Transection & 0 \\
Others (compression in the neck) & 1 \\
\hline
\end{tabular}

Table 3. Results of IONM

\begin{tabular}{lll}
\hline & \multicolumn{1}{c}{ Evaluation with IONM } \\
\cline { 2 - 3 } & \multicolumn{1}{c}{+} & \multicolumn{1}{c}{-} \\
\hline Motion of vocal cord (POD7) & & 2 \\
+ & 8 & Pseudo negative \\
- & & 45 \\
& 2 & \\
\hline
\end{tabular}

+ means loss of motion of vocal cord checked by ENT doctors or loss of response on IONM; - means no signs of paralysis checked by ENT doctors or adequate response on IONM. IONM: intra-operative neural monitoring; POD: postoperative day; ENT: ear-nose-throat. Sensitivity: $8 / 10=80 \%$; specificity: $45 / 47=95.7 \%$; positive predictive values: $8 / 10=80 \%$; negative predictive values: $45 / 47=95.7 \%$
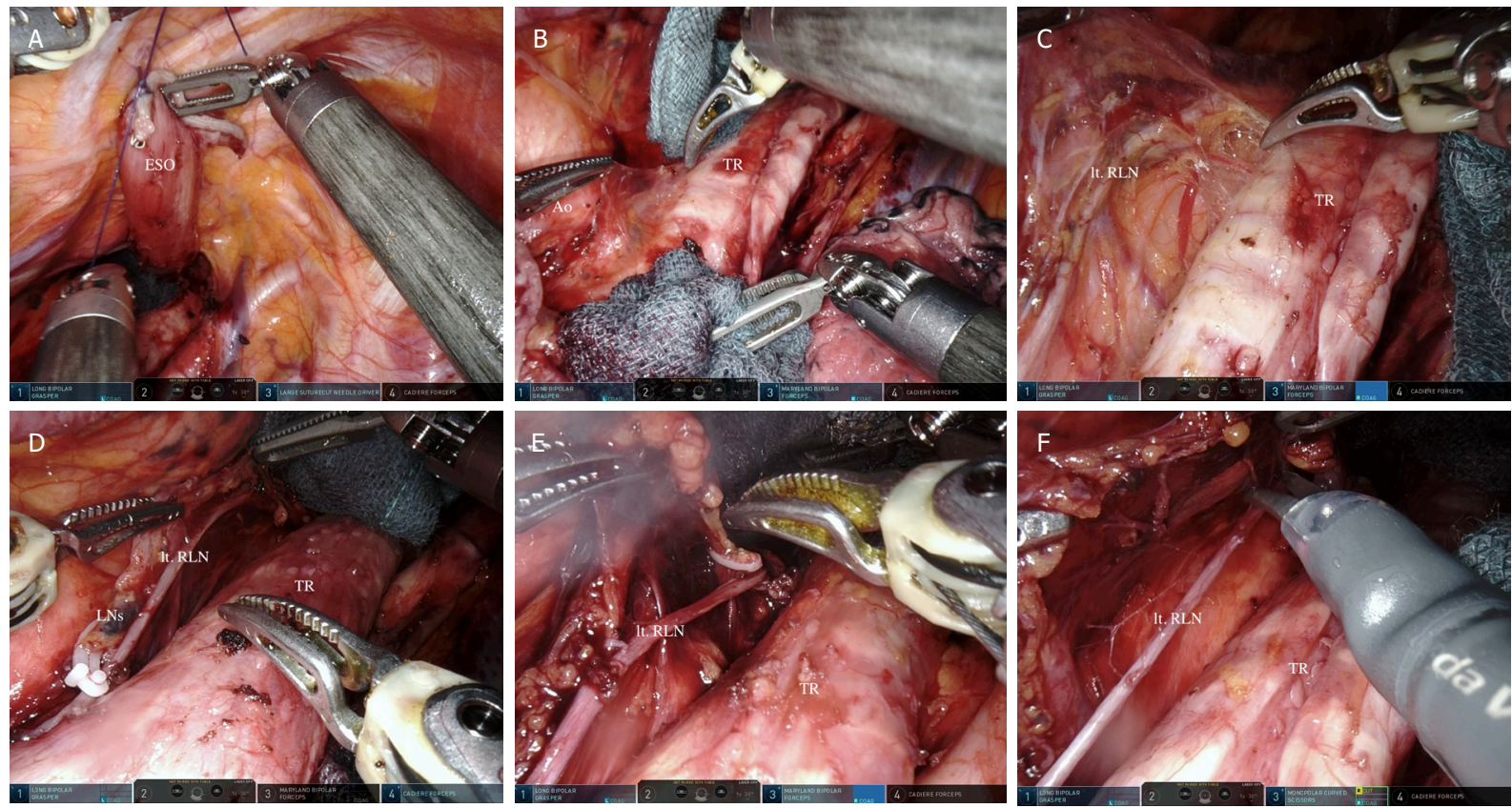

Figure 2. Dissection of lymph nodes around the left RLN. A: the esophagus was cut and the upper side mobilized and sewn to the chest wall; B: by compressing and rolling the trachea, we expanded the operative field of view on the left side and initiated lymph node dissection; C: using the glossy membrane as a landmark on the ventral side, we peeled off the adipose tissue including lymph nodes and proceeded cranially; D: the tissue to be dissected was peeled up behind the RLN; E: hemostasis was achieved by clipping the tracheoesophageal artery; F: connective tissue behind the RLN was cut to complete lymph node dissection around the left RLN. Ao: aorta; ESO: esophagus; TR: trachea; RLN: recurrent laryngeal nerve

tissue towards the dorsal side of the RLN and proceeded towards the cranial side. We clipped the dissected tissue at the most cranial side to achieve hemostasis of the tracheoesophageal artery [Figure 2E]. This clip demarcates the upper end of lymph node dissection from the thoracic cavity and served as a landmark for later dissection of lymph nodes in the neck. We also resected tissue on the dorsal side of the RLN [Figure $2 \mathrm{~F}$ ], which allowed dissection of lymph nodes around the RLN with minimal maneuvering of the nerve, thereby reducing the risk of RLNP. During the cervical procedure, attention to avoid nerve compression by the muscle retractor was necessary. 


\section{Short-term surgical results obtained using the modified surgical technique}

There were no differences between the conventional and modified surgery groups in terms of sex, age, body mass index, tumor histology and location, clinical stage, preoperative therapy, physical status, and other preoperative risks [Table 1]. None of the cases required conversion to open surgery and there was no intraoperative morbidity in either group. In the modified surgery group, the operation was performed robotically, which significantly shortened the procedure compared to the conventional surgery group (266 min vs. $295 \mathrm{~min}, P=0.04$ ). There was no significant difference in the estimated total volume of blood loss between groups, although it tended to be lower in the modified surgery group ( $91 \mathrm{~mL} v s .150 \mathrm{~mL}$, $P=0.08$ ). The number of dissected mediastinal lymph nodes was significantly higher in the modified surgery group ( 29 vs. $24, P=0.04$ ). There were also no differences in the rates of complications such as RLNP, aspiration, pneumonia, and anastomotic leakage between the two groups. However, the rate of RLNP tended to be lower in the modified surgery group than in the conventional surgery group ( $5 \% v s$. $17.5 \%)$. The median length of postoperative hospital stay was the same between groups (22 days).

\section{DISCUSSION}

RLNP is a relatively frequent complication of esophageal cancer surgery that affects the postoperative course and even overall survival ${ }^{[1,8]}$. There are several reports describing the effectiveness of IONM to prevent postoperative RLNP following esophagectomy ${ }^{[9-11]}$. At our hospital, the incidence of RLNP has declined since we introduced IONM but it has not been completely eliminated.

The surgical procedure(s) that lead to RLNP remain unclear, and there have not been reports to date addressing this point. In general, the cause was presumed to be either thermal injury from an energy device, or damage through nerve traction or compression. However, without identifying the cause, it is very difficult to implement effective preventive measures. On the other hand, IONM has long been used in the field of Otolaryngology in the treatment of thyroid cancer ${ }^{[12]}$ and there are many reports on the causes of RLNP after thyroid surgery ${ }^{[13-15]}$, with traction accounting for $75 \%-83 \%$ of cases. In this study, we found that RLNP following esophageal cancer surgery was similarly, primarily caused by traction and compression, with little contribution from thermal injury. This is the first report describing the causes of RLNP associated with esophageal cancer surgery, albeit in a small number of cases.

We have developed a modified surgical technique to prevent RLNP. Thermal injury occurred relatively early in the surgery and can be prevented by examining the location of the nerve by IONM. In recent years, mesentery-oriented lymph node dissection has become commonplace and has been proposed for esophagectomy ${ }^{[16]}$. Accordingly, we dissected the lymph nodes after mesenterization ${ }^{[6,8]}$. However, this inevitably increased the risk of strongly pulling the RLN, which could result in RLNP. We therefore concluded that it was difficult to prevent RLNP by this method (conventional surgery group, Figure 3).

The modified surgical technique is suitable for dissecting lymph nodes around the RLN with minimal retraction and compression and has in fact, reduced the rate of RLNP at our institution [Table 1]. Of the 20 surgeries performed after standardizing the procedure, there was only one case of RLNP in which the RLN was seized after misidentification, which counts as a technical error. There have been no instances of RLNP since due to an unidentified cause.

Robot-assisted minimal invasive surgery (Ra-MIE) was used in the modified surgery group for historical reasons. Approved as a medical treatment in Japan since 2018, Ra-MIE is advantageous in esophageal cancer surgery because it allows the operator to manipulate three arms in a stable field of view, even within a narrow space such as the upper mediastinum. Ra-MIE is particularly useful for the delicate manipulation required around the RLN. Thus, Ra-MIE undoubtedly contributed to the impressive results achieved with our modified surgical procedure. However, when comparing 33 Ra-MIE and 44 thoracoscopic surgeries, 

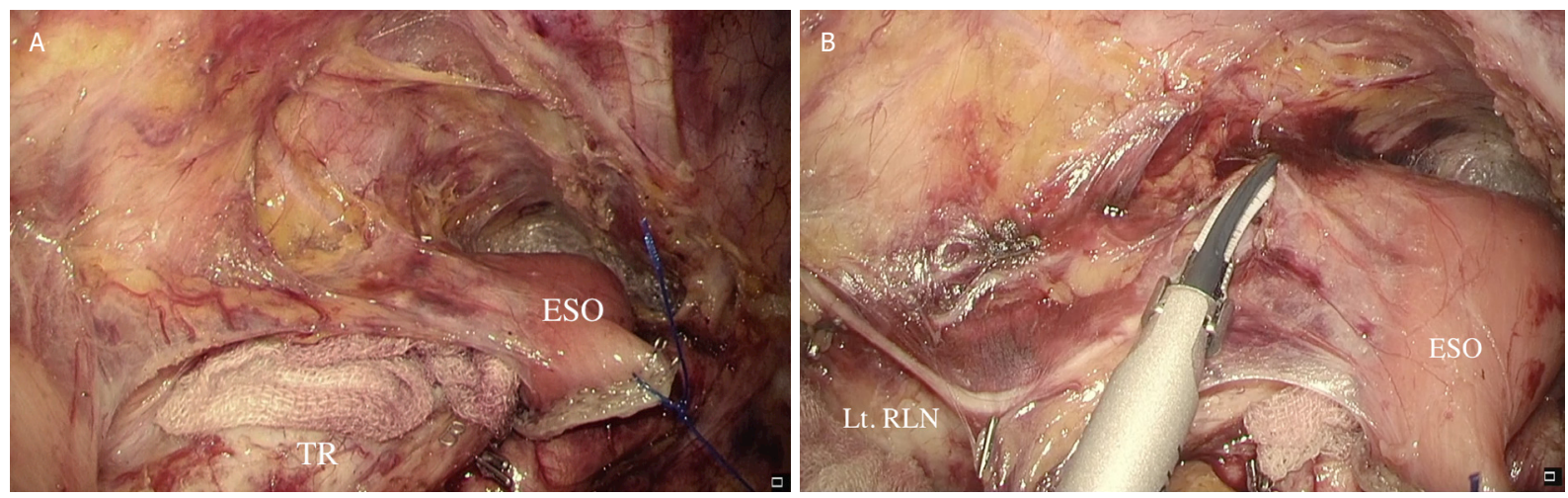

Figure 3. Lymph node dissection around the left RLN with the conventional technique. A: after dissecting the ventral and dorsal sides of the tissues which include the RLN, we divided the esophagus at the level of the aortic arch and drew the upper side of esophagus to the right to expand the operative field; B: the adipose tissue containing lymph nodes was resected while preserving the left RLN. Traction of the esophagus could flex the nerve or cause compression at the corner of the trachea. ESO: esophagus; TR: trachea; RLN: recurrent laryngeal nerve

the RLNP rates were $18 \%$ and $11 \%$, respectively (no significant difference). For this reason, we consider the modified surgical procedure to be more important in reducing RLNP.

There were some limitations in this study. First, the study was retrospective, single institution, and included only a small number of cases. Second, we only employed IONM intermittently whereas continuous monitoring may be more useful as it could provide a detailed view of nerve integrity in real time. Finally, our procedure does not allow en-bloc resection of lymph nodes surrounding the RLN, which could be considered as a shortcoming.

In conclusion, we demonstrated that the main causes of RLNP at our institution were due to compression and traction, not thermal injury. We also showed that our modified surgical technique can prevent left RLNP following upper mediastinal lymph node dissection in esophageal cancer surgery.

\section{DECLARATIONS}

\section{Acknowledgments}

We thank Ichiro Sasaki for technical assistance and advice on the NIM Nerve Monitoring system.

\section{Authors' contributions}

Designed the study and acquired, analyzed, interpreted of the data: Kobayashi $\mathrm{H}$

Contributed to data interpretation, reviewed the intellectual content of the manuscript, and approved the final version of the manuscript: Kobayashi H, Kondo M, Kita R, Hashida H, Shiokawa K, Iwaki K, Kambe H, Mizuno R, Kawarabayashi T, Sumi T, Kaihara S, Hosotani R

\section{Availability of data and materials}

Not applicable.

\section{Financial support and sponsorship}

None.

\section{Conflicts of interest}

All authors declared that there are no conflicts of interest.

\section{Ethical approval and consent to participate}

Not applicable. 


\section{Consent for publication}

Not applicable.

\section{Copyright}

(c) The Author(s) 2020.

\section{REFERENCES}

1. Noshiro H, Miyake S. Thoracoscopic esophagectomy using prone positioning. Ann Thorac Cardiovasc Surg 2013;19:399-408.

2. Takeuchi H, Miyata H, Ozawa S, Udagawa H, Osugi H, et al. Comparison of short-term outcomes between open and minimally invasive esophagectomy for esophageal cancer using a nationwide database in Japan. Ann Surg Oncol 2017;24:1821-7.

3. Sakamoto T, Fujiogi M, Matsui H, Fushimi K, Yasunaga H. Comparing perioperative mortality and morbidity of minimally invasive esophagectomy versus open esophagectomy for esophageal cancer: a nationwide retrospective analysis. Ann Surg 2019; Epub ahead of print. doi: 10.1097/SLA.0000000000003500.

4. Kataoka K, Takeuchi H, Mizusawa J, Igaki H, Ozawa S, et al. Prognostic impact of postoperative morbidity after esophagectomy for esophageal cancer: exploratory analysis of JCOG9907. Ann Surg 2017;265:1152-7.

5. Saeki H, Tsutsumi S, Tajiri H, Yukaya T, Tsutsumi R, et al. Prognostic significance of postoperative complications after curative resection for patients with esophageal squamous cell carcinoma. Ann Surg 2017;265:527-33.

6. Kobayashi H, Kondo M, Mizumoto M, Hashida H, Kaihara S, et al. Technique and surgical outcomes of mesenterization and intraoperative neural monitoring to reduce recurrent laryngeal nerve paralysis after thoracoscopic esophagectomy: a cohort study. Int J Surg 2018;56:301-6.

7. Rice TW, Ishwaran H, Blackstone EH, Hofstetter WL, Kelsen DP, et al. Recommendations for clinical staging (cTNM) of cancer of the esophagus and esophagogastric junction for the 8th edition AJCC/UICC staging manuals. Dis Esophagus 2016;29:913-9.

8. Oshikiri T, Yasuda T, Harada H, Goto H, Oyama M, et al. A new method (the "Bascule method") for lymphadenectomy along the left recurrent laryngeal nerve during prone esophagectomy for esophageal cancer. Surg Endosc 2015;29:2442-50.

9. Ikeda Y, Inoue T, Ogawa E, Horikawa M, Inaba T, et al. Recurrent laryngeal nerve monitoring during thoracoscopic esophagectomy. World J Surg 2014;38:897-901.

10. Tsang RK, Law S. Adaptation of continuous intraoperative vagus nerve stimulation for monitoring of recurrent laryngeal nerve during minimally invasive esophagectomy. World J Surg 2016;40:137-41.

11. Hikage M, Kamei T, Nakano T, Abe S, Katsura K, et al. Impact of routine recurrent laryngeal nerve monitoring in prone esophagectomy with mediastinal lymph node dissection. Surg Endosc 2017;31:2986-96.

12. Randolph GW, Dralle H, International Intraoperative Monitoring Study G, Abdullah H, Barczynski M, et al. Electrophysiologic recurrent laryngeal nerve monitoring during thyroid and parathyroid surgery: international standards guideline statement. Laryngoscope 2011;121 Suppl 1:S1-16.

13. Chiang FY, Lu IC, Kuo WR, Lee KW, Chang NC, et al. The mechanism of recurrent laryngeal nerve injury during thyroid surgery--the application of intraoperative neuromonitoring. Surgery 2008;143:743-9.

14. Phelan E, Schneider R, Lorenz K, Dralle H, Kamani D, et al. Continuous vagal IONM prevents recurrent laryngeal nerve paralysis by revealing initial EMG changes of impending neuropraxic injury: a prospective, multicenter study. Laryngoscope 2014;124:1498-505.

15. Schneider R, Randolph G, Dionigi G, Barczynski M, Chiang FY, et al. Prospective study of vocal fold function after loss of the neuromonitoring signal in thyroid surgery: The International Neural Monitoring Study Group's POLT study. Laryngoscope 2016;126:1260-6.

16. Shinohara H, Kurahashi Y, Haruta S, Ishida Y, Sasako M. Universalization of the operative strategy by systematic mesogastric excision for stomach cancer with that for total mesorectal excision and complete mesocolic excision colorectal counterparts. Ann Gastroenterol Surg 2018;2:28-36. 